Charlson Co-morbidity index $(2.5 \pm 0.7$ vs $2.3 \pm 0.6)$ were significantly increased in the delirium group. There were no significant differences between the groups in the use of neoadjuvant therapy. Analysis demonstrated that delirium was associated with a significantly longer hospital ( $14 \pm 7.5$ vs $10.9 \pm 5.7$ days) and ICU stay (3.6 \pm 3.8 vs $2.7 \pm 16.9$ days). Furthermore post-operative delirium was associated with a significantly increased incidence of postoperative pneumonia ( $21.7 \%$ vs $7.9 \%$ ), pneumothorax $(10.9 \%$ vs $2.6 \%$, re-intubation (10.9\% vs $1.8 \%$ ) and increased overall treatment costs ( $\$ 28223 \pm 13018$ vs $\$ 22702 \pm 9689 ; p<0.05)$. Age was the only pre-operative predictor of post-operative delirium in multivariate modelling (OR 1.08; 95\% CI 1.04 to $1.12, \mathrm{p}<0.05$ ). Patients were followed-up for an average of approximately 4 years. There was no significant difference between the groups in overall survival (1105 \pm 910 days vs $1273 \pm 1428 ; p=0.28)$.

Conclusion This study demonstrates that delirium is a risk factor for complicated post-operative recovery and increased treatment costs following oesophagectomy, and furthermore that age is independently predictive of its development. Focused screening will allow targeted preventative strategies to be employed in the peri-operative period to reduce complications and cost associated with delirium.

Competing interests None declared.

\section{PWE-035 CENTRALISATION OF UPPER GI CANCER SERVICES-IS THE HUB REALLY BETTER THAN THE SPOKE?}

doi:10.1136/gutjnl-2012-302514d.35

S Monkhouse, * J Torres-Grau, D Bawden, C Ross, R Krysztopik. Upper Gl Surgery, Royal United Hospital, Bath, UK

Introduction The aim of this study was to assess whether patients diagnosed with oesophageal or gastric cancer at a local district general hospital (the "spoke") have a similar temporal pathway through the decision making and treatment process compared to those patients presenting at the centralised, tertiary hospital (the "hub").

Methods Between April 2010 and April 2011, patients with a new diagnosis of oesophago-gastric cancer from both the hub and spoke hospitals were analysed. Data regarding diagnosis, time from diagnosis to multidisciplinary meeting (MDM) discussion and time from MDM decision to first treatment were all recorded. Statistical analysis was performed using parametric two-tailed t-test to assess significance.

Results There was a statistically significant increase in the time from diagnosis to MDM discussion at the spoke hospital compared to the hub (13.3 days vs +25.67 days; $\mathrm{p}=0.001$ ). However, time to first treatment (surgery, palliative therapy, neo-adjuvant therapy or best supportive care) was significantly increased in the hub hospital compared to the spoke ( 43.4 days vs 25.5 days; $p=0.023$ ).

Conclusion This study is the first of its kind to show that there is a disparity in the management pathways of patients who first present to a regional hospital rather than the tertiary centre. Patients at the spoke hospital have a longer lead time into the MDM but nonoperative treatment appears to be delivered more quickly locally.

Competing interests None declared.

\section{REFERENCES}

1. Polednak AP. "Trends in survival for both histologic types of esophageal cancer in US surveillance, epidemiology and end results areas". Int J Cancer 2003:105:98-100.

2. Birkmeyer JD, Stukel TA, Siewers AE, et al. Surgeon volume and operative mortality in the United States. N Engl J Med 2003:349:2117-27.

3. Gill AJ, Martin IG. Survival from upper gastrointestinal cancer in New Zealand: the effect of distance from a major hospital, socio-economic status, ethnicity, age and gender. ANZ J Surg 2002;72:643-6.

4. Department of Health. Improving Outcomes in Upper Gastrointestinal Cancers. London: Department of Health, 2001.
5. Siriwardena AK. Centralisation of upper gastrointestinal cancer surgery. Ann $R$ Coll Surg Engl 2007;89:335-6.

6. Siewert JR, Stein HJ. Carcinoma of the gastroesophageal junction: classification, pathology and extent of resection. Dis Esophagus 1986:9:173-82.

7. Mcleod U, Mitchell ED, Burgess C, et al. Risk factors for delayed presentation and referral of symptomatic cancer: evidence for common cancers. $\mathrm{Br} J$ Cancer 2009;101(Suppl 2):S92-101.

8. Forshaw MJ, Gossage JA, Stephens J, et al. Centralisation of oesophagogastric cancer services-can specialist units deliver? Ann $R$ Coll Surg Engl 2006:88:566-70

\section{PWE-036 WHAT IS THE SURVIVAL OF PATIENTS WITH OESOPHAGEAL CANCER FOLLOWING PALLIATIVE STENTING?}

doi:10.1136/gutjnl-2012-302514d.36

${ }^{1} \mathrm{~S}$ R Whiteoak, ${ }^{2} \mathrm{R}$ Frost, ${ }^{2} \mathrm{~J}$ Loehry, ${ }^{2} \mathrm{~K}$ Smith, ${ }^{2} \mathrm{~B}$ Colleypriest. ${ }^{1}$ MedicalGastroenterology, Poole General Hospital, Poole, UK; ${ }^{2}$ Medical-Gastroenterology, Salisbury District Hospital, Salisbury, UK

Introduction Oesophageal cancer is the seventh leading cause of cancer death worldwide. Unfortunately the majority of patients with oesophageal carcinoma are incurable at diagnosis. Selfexpanding metal stents (SEMs) are effective palliation for relieving dysphagia. The aim of this study was to determine survival duration following oesophageal stenting for malignant strictures and to identify potential factors that predict a poor outcome.

Methods We undertook a retrospective analysis to evaluate the outcome of patients following SEMs for malignant oesophageal strictures and possible prognostic factors over 6-year period (2004-2010). We analysed the Salisbury oesophageal stent database, reporting system and patient clinical management database to obtain data. We recorded age, sex, date of diagnosis stent and death, type of cancer, haemoglobin, creatinine, CRP and albumin, and whether chemotherapy or radiotherapy had been given. The results were statistically analysed using the unpaired t-Test and Pearson's correlation coefficient.

Results Between June 2004 and December 2010 we identified 128 patients who had one or more SEM inserted. One patient was excluded from the analysis. $46(36 \%)$ patients were female and 81 (64\%) male with a mean age of 76.7 (range 35-98). Adenocarcinoma accounted for 87 (69\%) patients, 38 (30\%) squamous cell carcinoma, and two others. The mean life expectancy was 147 days (range 8-1028 days) following the first stent deployment and 273 days (range 1-928 days) from diagnostic endoscopy. The 30-day mortality was $10 \%$. There was no difference in mortality when age $(p=0.19)$, sex $(p=0.35)$, haemoglobin $(p=0.23)$, CRP $(p=0.34)$, albumin $(p=0.36)$ or creatinine $(p=0.28)$ were compared. Patients with adenocarcinoma had a mean survival 163 days from initia stent which was statistically better than 108 days in the squamous cell carcinoma group $(\mathrm{p}=0.09)$. Patients receiving chemotherapy or chemoradiotherapy survived on average 18 days longer than those who had SEMs alone, regardless of histology $(p<0.0001)$

Conclusion Incurable oesophageal cancer has a bleak prognosis, but survival after SEMs is significant. In our study age, sex, and simple laboratory investigations were not predictive of mortality following SEMs. This suggests that it is not possible to estimate survival using any of these factors, and palliative SEMs should be considered in all patients. Squamous cell carcinoma has a significantly shorter life expectancy than adenocarcinoma following palliative SEMs. Those patients who had adjuvant chemotherapy or chemoradiotherapy had significantly improved survival, either due to the direct effect of the treatment or because of selection of fitter patients. Our data offers useful survival and 30-day mortality figures to help inform patients and make clinical management decisions.

Competing interests None declared. 